Voix et Images

\title{
Gabrielle Roy : la représentation du corps féminin
}

\section{Nicole Bourbonnais}

Volume 14, numéro 1 (39), automne 1988

France Théoret : narratrice de la subjectivité

URI : https://id.erudit.org/iderudit/200754ar

DOI : https://doi.org/10.7202/200754ar

Aller au sommaire du numéro

Éditeur(s)

Université du Québec à Montréal

ISSN

0318-9201 (imprimé)

1705-933X (numérique)

Découvrir la revue

Citer cet article

Bourbonnais, N. (1988). Gabrielle Roy : la représentation du corps féminin. Voix et Images, 14(1), 72-89. https://doi.org/10.7202/200754ar d'utilisation que vous pouvez consulter en ligne.

https://apropos.erudit.org/fr/usagers/politique-dutilisation/ 


\section{Gabrielle Roy: la représentation du corps féminin}

\section{par Nicole Bourbonnais, Université d'Ottawa}

Sinon comme en transit, de par son passage furtif dans les lieux obligés de la rhétorique conventionnelle, le corps féminin ne figure guère dans les romans québécois de la première moitié du $\mathrm{XX}^{\mathrm{e}}$ siècle. Quelques traits, toujours les mêmes, suffisent à peindre la figure féminine idéale, mère accomplie ou fille soumise: un corps robuste, des hanches fortes, des yeux clairs et doux; un sourire timide ou avenant, etc. Y a-t-il dérogation à cet usage, c'est qu'on bascule dans l'autre versant tout aussi conventionnel, le versant sombre et négatif de la femme dépravée, ce qui se reconnaît invariablement à l'aspect frivole de son corps: cheveux blonds mousseux, yeux effrontés, nez mutin, sourire enjôleur, etc. Même l'exception confirme la règle. La protagoniste du roman réaliste d'Albert Laberge, la Scouine, échappe certes au corps de la mère ou de la prostituée, mais c'est pour se voir refuser le droit à la féminité:

À seize ans, la Scouine était une grande fille, ou plutôt un grand garçon. Elle avait en effet la carrure, la taille, la figure, l' expression, les gestes, les manieres et la voix d' un homme.

L'hérö̈ne marginale paye de son corps la transgression qui consiste à déroger de la voie tracée aux femmes. Ainsi, régulièrement puisés à même un paradigme étroit de topoï descriptifs déterminés à l'avance, les matériaux constituant le corps féminin romanesque visent essentiellement un référentiel idéologique qui, au nom du bien collectif, occulte la dure réalité d'une vie de sacrifice et de servitude. De même qu'ils bloquent tout accès à une spécificité propre.

Pour la première fois, en 1945, avec le roman Bonheur d'occasion ${ }^{2}$, de Gabrielle Roy, 1'hypothèse de la vertu et du devoir qui grevait le corps romanesque est levée, mais c'est pour faire apparaître en pleine lumière l'insolvable du dénuement et de la misère qui se cachait sous les charges imposées par le système idéologique. Comme pour le Trente Arpents de Ringuet, la démarche adoptée est celle du réalisme, mais ici ce choix esthétique veut rendre ostensible non seulement la vie ingrate et misérable des défavorisés, mais aussi, et même surtout, l'injustice inséparable d'une existence de femme dont l'aire de

1 Alben Laberge, la Scouine, 3e édition, Montréal, l'Actuelle, 1972, p. 30.

2 Gabrielle Roy, Bonheur d'occasion, Montréal, Stanké, coll. «Québec 10/10m, 1977. Les citations empruntées à ce texte renverront à cette édition et les pages seront notées entre parenthèses. 
déplacement ne dépasse pas les limites du territoire occupé par les tâches de la reproduction et du maternage. En effet, si Ringuet contribue par son réalisme à démanteler l'image du paysan heureux dans une nature idyllique, il continue toutefois de souscrire à l'idée reçue de la femme admirable, quand elle est mère ou vouée à Dieu. Deux des filles d'Euchariste Moisan deviendront religieuses: il sera à peine parlé d'elles et encore moins de ce qui touche à leur chair. Par ailleurs, Lucinda, qui s'en va se perdre à la ville, est décrite en négatif, par le manque: elle n'avait pas ces calmes et doux yeux de brebis que l'on voit souvent aux filles de la terre, braves devant le travail et les maternités, mais craintives et timides devant l'étranger. ${ }^{3}$ La plus en chair sera, bien entendu, l'épouse et la mère, naturellement pourvue pour accomplir son destin de génitrice. N'a-t-elle pas la poitrine solide et généreuse, la bouche un peu lourde; les hanches larges oscillant avec un mouvement presque de berceau 4 ? La stratégie de Gabrielle Roy consistera à reprendre ces trois types consacrés - le corps effacé, le corps séducteur et le corps porteur - pour les déloger de la niche confortable qui les abritait, et les exhiber sous divers angles. Au lieu du commode et rapide signalement d'usage, elle détaille le corps avec insistance; au lieu de la représentation attendue, elle éclaire les ombres, les manques, les dessous, bref le réel de ce corps travesti. C'est principalement dans Bonheur d'occasion que s'exerce la très grande visibilité du corps féminin ${ }^{5}$. Dans les récits et romans qui suivront, ce corps sera, sinon évincé, du moins relégué à l'arrière-plan. L'abondance notable d'une part, la relative carence d'autre part, suscitent l'interrogation et invitent à l'analyse.

Une lecture rapide et superficielle de Bonheur d'occasion peut facilement induire en erreur et porter à croire que chacune des deux protagonistes bénéficie d'un statut corporel particulier. Mais cette conclusion ne résiste pas à une analyse plus approfondie. En effet, comme nous le verrons, le portrait des deux femmes s'élabore à partir d'un code sexuel et social qui les réunit dans un même destin et les dépersonnalise ${ }^{6}$. Avec comme troisième et dernier terme l'austère figure de la vieille Mme Laplante, la perte d'identité est accomplie. Celle qui a

3 Ringuet, Trente Arpents, Montréal et Paris, Fides, 1938, p. 167.

4 Ibid., p. 27.

5 Voir la concordance de Bonheur d'occasion établie par Paul G. Socken, Toronto, University of Waterloo Press, 1982, 1136 pages. La liste des fréquences permet de constater que les éléments corporels occupent la place la plus importante après les déterminants, pronơns, mots charnières, etc.

6 Lecteurs et critiques ont mis du temps à comprendre que ni Florentine ni Rose-Anna n'avaient une existence propre qui ferait de l'une d'elles la protagoniste du roman, mais qu'ensemble, elles incarnaient la condition féminine. C'est André Brochu qui, l'un des premiers, dans une pénétrante étude intitulée aThèmes et structures de Bonheur d'occasion» (publiée dans I'Instance critique, 1961-1973, Montréal, Leméac, 1974, p. 206-246), a vu que Florentine et Rose-Anna étaient unies par leur destin commun, legs héréditaire de la misère et de la maternité (p. 233). On peut toutefois déplorer que Brochu n'ait pas approfondi sa réflexion sur la condition féminine. Par exemple, parler de la possessivité - la part de ematernalisme» inscrite en Florentine par l'hérédité toute puissante (p. 237) revient presque à renvoyer le problème au seul personnage où à en situer la source dans l'hérédité, c'est-à-dire encore une fois dans la nature féminine ou le destin biologique. 
pourtant eu quinze petits corps accrochés d ses jupes, quinze petites têtes rondes et lisses entre son sein (p. 198), n'a jamais joui de la proximité de leur chaleur pas plus qu'elle n'a été touchée par leur grâce. Dépouillée de toute présence charnelle avec des doigts secs, jaunis (p. 199), son froid visage blanc et anguleux, sa petite voix pointue (p. 197), elle n'est plus que pur symbole chargé de représenter l'inflexible loi du fardeau de l'existence que nulle jouissance ne vient alléger. Et, de fait, pour ces trois corps dont l'expérience déterminante est celle de la grossesse, soit du poids à porter, il n'est pas question du plaisir obtenu par d'affectueuses embrassades, encore moins par de plus vives voluptés. De la sorte, quelque part entre le discours établi et cette nouvelle mise en scène romanesque, un grincement de désaccord se fait entendre. A disparu l'image consacrée de la mère plantureuse et rayonnante, à la chair épanouie. Et cela, qu'elle soit de la campagne ou de la ville.

L'articulation syntagmatique révèle aussi cette fusion de trois femmes en une. Il n'est pas indifférent que le personnage du roman soit celui de Florentine, qui est en attente du mariage et, obligatoirement, de la maternité, de même que ce n'est pas un hasard si Rose-Anna n'apparaît qu'au cinquième chapitre, vivante incarnation de l'avenir de Florentine, surgissant au moment stratégique où le corps aguichant de la fille va rejoindre celui de la mère. Puis, enfoui au centre du roman, le corps désormais inutile de la grand-mère complète la trajectoire du destin féminin. Enfouissement qui est aussi synonyme d'enracinement: l'image de la vieille mère est toujours déjà là, indélogeable, incrustée dans l'imaginaire de Rose-Anna dont l'image, à son tour, hante l'esprit de la fille. Le cercle est infini, infernal. Il devient vite évident qu'aucun des programmes narratifs des personnages féminins ne s'explique sans ce passage obligatoire par l'assujettissement au corps. En fin de compte, c'est lui le véritable protagoniste du roman.

\section{La promise au corps fictif}

Le premier corps à être mis en scène sera donc celui de la jeune fille à marier. Le début du roman coïncide avec les débuts de Florentine qui se met à exister à partir du moment où ses attraits physiques sont ouvertement reconnus par un homme:

A cette heure, Florentine s'était prise à guetter la venue du jeune homme qui la veille, entre tant de propos railleurs, lui avait laissé entendre qu'il la trouvait jolie. (p. 11)

La valeur marchande de son capital de séduction ayant été reconnue, elle peut espérer mener à bonne fin cette complexe transaction qu'est le mariage:

De toutes les pensées confuses qui avaient traversé son esprit, elle ne gardait qu' une impression nette, âpre comme son sourire figé: c'est $q u ' i l$ fallait jouer maintenant, immédiatement, tout ce qu'elle était encore, tout son charme physique dans un terrible enjeu pour le bonheur. (p. 20) 
Le conditionnement est si achevé que la jeune serveuse n'a même pas conscience de se définir par son corps et d'assigner à son existence les limites temporelles de la beauté: tout ce qu'elle était encore (p. 20). Or, paradoxalement, ce corpscapital souffre d'un manque d'investissements personnels. La charge modalisatrice des énoncés désigne clairement la passivité et la vacuité du visage au sourire vide, taciturne et morose qui s'accrochait sans but d quelque objet chatoyant qu' elle ne voyait pas (p. 11). Tel un sismographe, le corps reçoit et enregistre passivement les impressions reçues; il est si perméable qu'il se coule dans l'atmosphère artificielle qui l'entoure. Le regard fuit dans le miroitement de la verroterie, des panneaux nickelés, de la ferblanterie (p. 11). En quête d'un signifiant par quoi se représenter et se soutenir, le vide ne trouve que faux décor et objets de pacotille. Le texte fait de nouveau intervenir le maître-signifiant, soit la figure masculine. Pour la tirer de cet état à la fois léthargique et hystérique qui est le sien, de ce sommeil lourd où elle avait été blottie (p. 15), il lui faut être mis en présence de qui incarne le savoir et le pouvoir:

C'était lui [Jean] qui avait donné une expression d ces espoirs qui étaient maintenant aigus, torturants comme de l'envie. (p. 15)

On ne peut plus clairement signifier que la conscience du moi vient d'une scène qui lui échappe et que d'autres seront chargés d'appréhender à sa place.

La description de ce corps incontoumable passe par la focalisation multiple, technique qui permet de créer un effet de dissolution du sujet regardé. C'est ainsi que la vérité corporelle de Florentine apparaît problématique, car elle est soumise au croisement contradictoire des perceptions. Trois grands centres focalisateurs président à la mise en texte du corps de Florentine, faisant relever celui-ci moins d'une hypothétique réalité que d'un découpage en trois grandes zones du code descriptif conventionnel, qui se voit ainsi forcé de sortir de son cadre rassurant pour laisser paraître ses failles et ses contradictions. De ce corps sans grande cohérence, sans unité immédiatement repérable, se remarquent: 1) d'une part, l'irrésistible pouvoir de séduction tel que vu/imaginé par l'œil myope de Florentine; 2) d'autre part, l'allure enfantine et vulnérable que retient le point de vue extérieur; 3) et, enfin, la maigreur extrême qui ne peut échapper au regard inquisiteur de Jean. Ce dernier tient lieu d'agent révélateur tandis qu'à l'opposé, celui de Florentine est un non-regard, seul sensible aux fictions intériorisées par l'esprit. Le manque flagrant comme l'excès brutal se trouvent être réconciliés par le regard d'indulgence et d'intelligente compréhension qui émane autant du point de vue extra-diégétique que de cette incarnation romanesque de la pitié universelle qu'est Emmanuel.

Plus d'une fois, Gabrielle Roy choisit de représenter le personnage de Florentine dans des scènes d'auto-contemplation devant le miroir. Cette glace tendue, qui fonctionne comme un miroir aux alouettes, permet de faire ressortir la tragique inconscience de celles qui puisent leurs modèles culturels dans des romans-feuilletons de quinze cents (p. 14). 
Puis elle s'examina, satisfaite, et se vit pâle malgré le rouge mais jolie, plus jolie que jamais avec ses cheveux dénoués et ses yeux que la crainte avait agrandis. Elle étudia sa taille fine comme si jamais encore elle ne l' avait bien remarquée. Elle fit briller ses cheveux dans la glace au-dessus de la lampe d'applique, elle étendit ses mains devant elle, considéra ses doigts fins, ses ongles teintés de carmin, et devant sa jeunesse, ses cheveux épars, la blancheur de ses bras, elle recommença d'aimer la vie. (p. 257)

Il ne s'agit pas d'un regard anodin, jeté à la dérobée, ou d'une innocente contemplation mais bien d'une étude délibérée. Non point utilisée pour authentifier l'autoportrait, mais plutôt comme icône de la contemplatrice absorbée par son reflet, le miroir est à la fois surface et simulacre sur lesquels se fixent amoureusement les yeux envoûtés par le sortilège du joli tableau que composent la taille fine, les cheveux dénoués, les doigts fins, la blancheur des bras, etc. Rien de personnel ni de spécifique dans ce portrait où toute individualité est gommée. L'effet déréalisant des images à la mode est chaque fois tout aussi efficace et tout aussi nocif. Au restaurant, en compagnie de Jean, Florentine, le regard caractéristiquement «perdu dans le vague», se noie irrémédiablement:

Ce qui la grisait surtout, c'était dans une glace profonde derrière le jeune homme, sa propre image vers laquelle elle se penchait fréquemment. Elle s'y voyait, les yeux brillants, le teint mat et clair, les traits dilués; et parce qu' elle se plaisait ainsi, elle avait l'air en s'approchant de Jean de vouloir sans cesse lui communiquer son instant de triomphe [...].

Elle ne voyait plus qu'il l'écoutait à peine et la regardait à la dérobée avec ennui. Elle se parlait plutôt à elle-même, ployant le buste vers les yeux de flamme qui l' encourageaient, qui la soutenaient, qui la grisaient du fond de la glace. (p. 83-84)

Le repliement narcissique sur la vision de rêve conduit à la perte de l'autre comme à la déperdition de soi.

Dans les scènes du miroir se rejoue à chaque fois, au féminin, le drame de Narcisse, mais conjugué à celui d'Écho, car Florentine, en s'adressant intérieurement des compliments, ne peut que répéter les mots passe-partout qui servent à décrire l'idéal esthétique des photos-romans et feuilletons de quinze cents. Ce n'est pas seulement l'emprise néfaste d'une sous-littérature d'occasion qui est ici accusée, mais aussi, implicitement, par le manque, l'impuissance de la bonne littérature à fournir des modèles crédibles et valables de la réalité féminine. Car, pas plus que la jeune citadine ne possède le doux regard timide de la vertueuse fille de campagne ou la coquetterie perverse de la fille perdue, pas plus le corps anguleux de la vieille Mme Laplante ou celui, déformé, de Rose-Anna ne correspondent au corps épanoui et généreux de la mère idéale. Est aussi posée la question du destinataire. À qui au juste s'adressaient les romans de la terre? On n'imagine aucun des personnages féminins de Bonheur d'occasion les lisant. 
Pour délivrer lecteurs et lectrices de ces fictions, il fallait leur opposer une vision réaliste. Gabrielle Roy crée un personnage d'homme d'action, opportuniste et ambitieux, peu enclin à l'idéalisation, dont le regard décapant aura vite fait de réduire le corps féminin à sa vérité la plus stricte. La glace qui, pour le personnage féminin, faisait office de voile et de leurre, devient, mise au service du personnage masculin, un instrument de traversée des apparences. Le regard emprunte le détour de la glace, ce qui lui permet de voir sans être vu, à l'insu de la victime qui ne songe donc pas à brouiller son image. Ainsi s'institue un double rapport à la réalité, l'un de clairvoyance, l'autre d'aveuglement:

Les coudes au comptoir, les yeux rivés à ceux de Florentine, il attendait...

Elle se raidit sous ce brutal examen, et il la vit mieux; il la vit reflétée d mi-corps dans la glace du mur, et il fut frappé de sa maigreur. Autour de sa taille, elle avait pourtant tiré jusqu' au bout le ceinturon de son uniforme vert, mais on devinait que ses vêtements adhéraient à peine à son corps fluet. Et le jeune homme eut soudain une vision de ce qui pouvait être sa vie, [...] cette vie des jeunes filles fardées, pimpantes, qui lisent des romans-feuilletons de quinze cents [...].

Il remarqua le tressaillement de ses mains, frêles comme celle d'un enfant, il vit les clavicules se découper dans l'échancrure du corsage. (p. 14)

Le pouvoir de séduction semble s'être envolé pour être entièrement supplanté par cette découverte stupéfiante: la maigreur, totalement définissante de la jeune fille. Ce bref espace textuel est saturé de termes connotant son décharnement. D'ailleurs, nulle mention du visage dans cette description «à mi-corps» où se trouve surtout retenu ce qui fait squelette: les clavicules, les mains frêles, le corps fluet. En outre, l'insistance du texte à faire valoir la puissance scopique du sujet masculin a pour effet de diminuer encore davantage l'objet regardé. Même lorsque la maigreur de la jeune fille est camouflée par de pitoyables artifices, elle n'échappe pas à l'œil radiographique, et, dès lors, elle se métaphorise en un dénuement total de l'être:

[...J mais il ne la voyait plus telle qu'elle était là de l'autre côté du comptoir. Il la voyait parée, prête à sortir le soir, avec beaucoup de fard pour couvrir la pâleur de ses joues, des bijoux cliquetant sur toute sa maigre personne, un petit chapeau ridicule, peut-être même une voilette derrière laquelle ses yeux avivés de khôl brilleraient [...]. (p. 15)

Ce corps chosifié, déjà ravi à lui-même, ne peut avoir qu'une fin: le rapt, soit par le séducteur qui en jouit, soit par la couvée qui se l'approprie. Dès le premier tête-à-tête bien inégal entre elle et lui - scène type qui se répétera à chacune de leurs rencontres -, il est évident que le rapport du masculin au féminin est celui du prédateur et de sa proie: 
Les coudes au comptoir, les yeux rivés a ceux de Florentine, il attendait maintenant d'elle, comme dans un jeu cruel, avec patience, un premier mouvement sur lequel il réglerait le sien. (p. 14)

D'emblée, par le regard, est signifiée la destination de ce corps mal établi, le viol dont il est/sera l'objet. Dans ce jeu cruel, aux règles strictes, la manœuvre du partenaire féminin est d'avance condamnée à l'échec. En montrant les règles du jeu, Gabrielle Roy en éclaire les vices cachés.

La réification du corps féminin se poursuit sous le regard de l'instance narrative qui corrobore pour l'essentiel les observations de Jean dont la crédibilité se trouve ainsi rehaussée. Un inventaire rapide des expressions utilisées pour la description de Florentine produit un lexique qui pivote autour de l'état de privation. Alors que dans le vocabulaire prêté à Jean, le substantif «maigreur» et ses dérivés sont privilégiés, au sein du lexique retenu par la voix anonyme, plus adonnée à la commisération, se marquent des expressions euphémisantes comme «menu», «délicat», «fluet». Quasi invariablement, la déléguée à la narration donne à voir un corps fragile et pitoyable: mains qui tressaillent, pas chancelants, sourire lamentable ou crispé, yeux tourmentés ou fuyants. Le recours fréquent et délibéré au qualificatif «petit», s'il suggère au lecteur une attitude de compassion, tend aussi à diminuer dans son esprit les capacités de la jeune fille, à l'infantiliser: son petit nez frémissait (p. 78), une petite main nue (p. 78), un petit ongle mis d nu (p. 82), une petite tête ronde et lisse (p. 135), etc. Sans maîtrise, déconnecté du libre arbitre, le corps de Florentine est agi plutôt qu'il n'agit. Il est le corps-lige par excellence.

La distance incommensurable qui sépare l'image du corps telle que rêvée par Florentine et la réalité corporelle qui est découverte par les deux regards les plus dignes de foi témoigne bien du clivage profond qui paralyse tout son être. Non seulement les modeles culturels bloquent-ils l'accès au corps propre, non seulement les mêmes images saintes, les mêmes portraits de famille viennent-ils se placer devant elle comme pour l'emprisonner (p. 254), mais ils la rendent aveugle au rapport existant entre le corps séducteur et le corps reproducteur.

Dans le roman Trente Arpents, emblématique des préjugés tenaces qui ont servi à définir la femme pendant longtemps, jamais la moindre confusion n'estelle possible entre le corps-objet, offert a la convoitise sexuelle et le corps procréateur, objet du désir de perpétuer la race. Le corps désirable de Phonsine que devinait goulûment [le] désir d' homme d'Euchariste Moisan est indissociable du corps maternel à la poitrine généreuse, aux hanches larges oscillant avec un mouvement presque de berceau. ${ }^{7} \mathrm{~L}$ 'adverbe "goulûment» n'est pas sans rappeler la tétée du nourrisson. De même, la sémiologie du corps de Lucinda, habillé d'une robe de taffetas vert sans manches qui offrait la chair appétissante de ses bras $n u s^{8}$ est aisément décryptable. Mais dans Bonheur d'occasion, ce système clos, 
aux catégories bien étanches, est contrecarré par un brouillage qui fait se désaccorder signifiants et signifiés, court-circuitant les données d'arrivée. Florentine joue de la séduction de ses charmes sans pourtant jamais penser à les monnayer comme Lucinda; d'autre part, elle oublie la fonction reproductrice liée à cette séduction. Tout se passe comme si, avec sa maigreur, elle se croyait à l'abri de la procréation que seules les réglementaires hanches larges permettraient d'imaginer.

Ce divorce est particulièrement manifeste dans la scène où Jean lui rend visite en l'absence de ses parents:

Des bijoux de couleur vive sautaient d̀ son cou et d̀ ses bras, s'entrechoquaient et semblaient exprimer sa volonté nerveuse; mais sur sa robe de soie noire un petit tablier de caoutchouc glissait d̀ ses hanches, d̀ chaque mouvement de son corps, avec un bruit doux et continu. (p. 203)

Ce portrait de Florentine représente bien un moi éclaté qui se disperse dans des directions contradictoires: la domesticité, la coquetterie, l'assurance et la soumission. L'issue prévisible se produit: le corps divisé craque, la bretelle $d u$ tablier qui saute (p. 209) devient la synecdoque du corps de la ménagère mal articulé au corps de la séductrice, qui se retrouve en posture précaire, symptomatique de tout son être: tombée à la renverse, les genoux repliés un petit pied battant l'air (p. 210).

Jamais à sa place, sans cesse disqualifié, le corps féminin se retrouve sans racines, sans attaches, exilé de son espace naturel. Il s'agit, à mon avis, de plus que de la simple transplantation des Lacasse en ville ou des effets de la pauvreté. Le corps apprêté que Florentine exhibe si naïvement n'a-t-il pas, avant tout, été déporté de la nature vers la culture? Ou plutôt du naturel vers l'artificiel? Une déportation qui fait de lui ce corps endimanché que Florentine promène au bord du fleuve vêtu comme pour la ville, avec des gants, des talons hauts et une petite toque toute couverte de verdure et de fleurs raides (p. 329). Ou encore cette maigreur parée et masquée par les fards, les bijoux, les colifichets. Le maquillage, la parure, les artifices qui dénaturent Florentine pourraient bien composer le scénario qui viendrait confirmer l'énoncé suivant tiré du Deuxième Sexe:

Le rôle de la parure est à la fois de la [la femme] faire participer plus intimement d̀ la nature et de l'en arracher, c'est de prêter à la vie palpitante la nécessité figée de l' artificiel. ${ }^{9}$

Ou qui viendrait appuyer le point de vue de Madeleine Ouellette-Michalska:

Le maquillage-repliement narcissique de la femme sur son propre corps? Ou comme une soumission au discours patriarcal qui, traditionnellement, ne lui ouvrait l'accès au champ social que par le biais de la séduction et de la procréation ? $^{10}$

$9 \quad$ Simone de Beauvoir, le Deuxième Sexe, tome 1, Paris, Gallimard, 1949, p. 218.

10 Madeleine Ouellette-Michalska, l'Echappée des discours de l'Cil, Montréal, Nouvelle Optique, 1981, p. 132. 
La litigieuse opposition nature-culture, tant de fois débattue par les féministes contemporaines, se trouve déjà scénarisée dans Bonheur d'occasion, où celle qui interprète le rôle de la jeune fille est exilée de la nature pour ensuite être contrainte d'en retrouver le difficile chemin à cause d'une grossesse forcée. Pourtant, derrière ces contraintes et ces refoulements, il arrive que se devine ou s'échappe, toujours à la dérobée, le désir ou la volonté propre. Jean n'est-il pas soudain frappé par l'expression d'avidité qu'il découvre sous les paupières baissées: [...] les paupières en s'abaissant ne livraient qu' un mince rayon de regard mordoré, prudent, attentif et extraordinairement avide (p. 13). Lors de la soirée chez Emmanuel, c'est le désir qui se manifeste dans l'abandon du corps au rythme de la danse ainsi que dans le libre mouvement des cheveux flottants. Et Rose-Anna ne voit-elle pas juste lorsqu'elle remarque soudain que sa fille mince et hardie [...] avait l' air d' un garçon (p. 107)? Ainsi, l'auteure en introduisant ces brèves échappées de vérité et de naturel, s'emploie à replacer ce qui avait été déplacé par un discours faux et contraignant.

\section{Un encombrant embonpoint}

Si paradoxal que cela puisse sembler, du sein de cette scène corporelle aberrante et contradictoire, émerge un noyau permanent, indissoluble, qui dote la protagoniste inconsistante d'un semblant de définition et, surtout, d'une volonté d'émancipation et d'affirmation de soi. En effet, si le jeu de la focalisation multiple, avec ses écarts et ses recoupements, contribue à rendre manifeste l'incohérence du corps féminin, il atteint encore un autre résultat qui se situe presque aux antipodes du premier: au sein de leurs divergences respectives, les principaux centres focalisateurs convergent vers une constante, l'extrême minceur de Florentine, qui finit de la sorte par constituer sa véritable identité. Voilà que le décharnement physique et moral de Florentine, d'une part si dévalorisant, se trouve composer le trait qui la particularise et l'individualise.

Car c'est bien grâce à sa maigreur que Florentine se distingue des autres personnages féminins du roman. Des cinq autres jeunes serveuses par exemple, condensées en la personne de la grosse Marguerite (p. 19) comme l'appelle dédaigneusement Florentine. L'instance focalisatrice externe confirme ce trait anatomique lorsqu'elle décrit Marguerite comme une grosse et grande fille (p. 18). II n'est pas jusqu'au pitoyable Alphonse, l'ami de Marguerite, qui ne la traite de bonne grosse fille, la Guitte (p. 308). Tous lui reconnaissent comme principale caractéristique cette chair plantureuse qui la destine tout «naturellement» à la fonction reproductrice. D'ailleurs, n'est-elle pas l'incarnation de la nature? Paysanne, avec ses joues sans fard, naturellement roses, son rire gras et bienveillant, sa lèvre humide (p. 18-19), elle fonctionne comme l'image inversée de la maigre citadine, fardée et cirsconspecte. De surcroît, un réseau de cellules narratives tissent autour d'elle la toile de la maternité. Le mari qui lui est dévolu est le type du parfait parasite, cet Alphonse dont même l'armée ne veut pas, et qu'elle, en mère indulgente et bonne, nourrit, habille et entretient. Au restaurant, elle n'est jamais impatiente, mais toujours souriante, accueillante avec les clients. C'est encore en mère compréhensive et dévouée qu'elle se comporte avec l'infortunée 
Florentine, venue se réfugier chez elle immédiatement après avoir été rejetée par Rose-Anna. Substitution éloquente à tout le moins! Marguerite éprouvait surtout le désir de la couvrir, de la protéger (p. 268), et pour l'amener à se confier, agit avec elle comme on fait avec les enfants pour solliciter un aveu (p. 267). Ainsi, d'une unité narrative à l'autre, la grosse et grande fille est-elle progressivement dépouillée de toute personnalité propre pour être entièrement revêtue des attributs maternels auxquels elle s'identifie totalement.

Mais, au contraire de ce qui se passe dans les romans antérieurs, elle n'est ni glorifiée ni proposée comme modèle. La stratégie ici consiste plutôt à transmuer le modèle consacré de la mère admirable en anti-modèle, voire en caricature. Le qualificatif «grosse» tant de fois répété finit par prendre une teinte péjorative. Et le dévouement maternel de Marguerite n'est ni efficace ni valorisant. D'une part, Florentine repousse son offre d'aide, presque avec violence, comme si elle ne pouvait plus supporter cette absurdité qui lui fait trouver la compréhension maternelle là où elle ne s'y attend pas et ne veut pas la voir tandis qu'elle lui fait défaut là où elle devrait être. D'autre part, le dévouement pour l'irrécupérable Alphonse est à la fois vain, naïf et navrant et, par là, le personnage de Marguerite est un double mineur de Rose-Anna qui se sacrifie aussi pour son mari et sa famille, sans jamais obtenir les résultats escomptés. Le personnage épisodique de Marguerite revêt donc une importance capitale dans la mesure où, de par sa fonction double et de contre-modèle, il déconstruit le paradigme traditionnel de la mère idéale pour édifier celui, plus réaliste, de la mère inefficace et aliénée.

Dans la catégorie des grosses et des mères entrent aussi la corpulente Emma Philibert, grosse, ronde, et pesante (p. 52), familièrement appelée maman Philibert par les jeunes chômeurs du quartier, la douce Mme Létourneau, toute ronde ((p. 130) qui est plus la mère de son mari que son épouse et enfin et surtout, la si maternelle Rose-Anna qui semblait toujours porter un fardeau sous la robe gonflée (p. 89). Ces femmes s'inscrivent dans la série paradigmatique des autres accoucheuses de l'cuvre de Gabrielle Roy. Qu'on songe en particulier à la courageuse Luzina de la Petite Poule d'eau qui, chaque année, régulièrement, met un enfant au monde: c'est une femme assez forte, grosse aux jambes enflées ${ }^{11}$, à la poitrine forte. Parfois, l'épaisseur des vêtements remplace la chair: c'est le cas de Thérésina Veilleux, dans Rue Deschambault ${ }^{12}$, qui vit en recluse dans sa chambre sous une montagne de vêtements qui lui font un corps volumineux, tout en la privant d'un corps propre. Or, la seule activité de ce personnage est de mettre périodiquement des enfants au monde, dont elle ne s'occupe plus par la suite. Le qualificatif «grosse» constamment associé au personnage de la mère, assimile cette demière au ventre reproducteur et l'identifie à la matière brute. Elle est grosse, c'est-à-dire perpétuellement assujettie à l'état de grossesse.

Constantes et contrastes investissent le défilé des mères grosses, d'un sens qui va au-delà du simple réalisme. Est-ce pur hasard si, du côté des maigres,

11 Gabrielle Roy, la Petite Poule d'eau, Montréal, Beauchemin, 1960, p. 16. 
figurent les récalcitrantes à la loi biologique? Telle la petite Yvonne qui, à peine âgée de 13 ans, se destine déjà à la vie religieuse, décision qui n'est pas sans rapport avec la pitié profonde qu'elle éprouve pour sa mère. Or, il se trouve qu'elle est pourvue d'un corps tout à fait en conformité avec son futur état de vierge inféconde. Elle se tient raide, le corps rigide, avec des bras grêles et une poitrine grêle (p. 353). Ou encore elle est décrite comme une fillette chétive dont la robe étriquée battait, étroite et longue sur ses jambes minces (p. 359). Il ne s'agit pas de désavouer la fonction de cette description comme signe de pauvreté, mais bien d'y indexer les éléments indiciels de la condition féminine. Car c'est bien le corps tout entier qui est nié par la fillette alors qu'elle le néglige, ne se soucie pas de sa tenue ou encore le maltraite en se rendant à l'église par n'importe quel temps, sans être chaudement vêtue. D'ailleurs, lorsque dans des œuvres ultérieures, auront disparu les manifestations de la misère, vont se maintenir celles d'un refus du corps. À titre d'exemple, mentionnons le personnage d'Odette dans Rue Deschambault dont le corps, soigneusement occulté, n'est représenté que par les costumes excentriques qui le déguisent comme le fera plus tard le costume de la religieuse. Il y a aussi Marie, la scur d'Emmanuel, au nom bien caractéristique, qui, toujours pâle, fatiguée, fait partie des ombres au corps effacé. Telle une morte, elle ne connaît pas le rire libérateur et la gaîté tonifiante. Il ne peut être question de mariage pour cet être diaphane si peu charnel, si peu terrestre. Participent d'une nature analogue les deux scurs marginales de la narratrice dans Rue Deschambault: Agnès, de constitution fragile, emprunte la voie de l'abnégation tandis qu'Alicia, au fin visage pâle ${ }^{13}$, finit par sombrer dans l'aliénation; elle aura un corps tout mou ${ }^{14}$, comme rompu. Tout se passe comme si Gabrielle Roy vouait ses jeunes personnages féminins à une extrême émaciation, jusqu'au point de disparition de leur corps problématique.

\section{Une maigreur signifiante}

D'entre toutes les jeunes créatures fictives de Gabrielle Roy, Florentine est la plus élaborée et la plus exemplaire. Sa maigreur se conjugue parfaitement avec sa nostalgie répétée d'une vie où la programmation biologique du corps féminin ne viendrait pas contrecarrer son désir d'épanouissement personnel. Ne se prometelle pas de ne jamais accomplir le long voyage gris, terne de sa mère (p. 120)? Et un sentiment de colère ne l'envahit-elle pas lorsqu'elle apprend que sa mère est de nouveau enceinte? La sexualité lui apparaîtra comme un piège $[. .$.$] tendu à sa$ faiblesse et qui lui fait éprouver un indicible mépris pour sa condition de femme (p. 253). On pourrait imaginer un scénario selon lequel, le premier mouvement de peur et de révolte passé, la fille enceinte, dans un juste retour vers la nature, serait émue à l'idée de l'enfant à naître et qu'elle porte dans son sein. Or, il n'en est rien. Jamais Gabrielle Roy ne la pourvoit d'un quelconque instinct maternel. C'est bien plutôt dans le sens d'une dénégation totale de la fonction reproductrice que peut s'interpréter l'insistance du texte à traduire le rejet obstiné du ventre comme l'obsession de la minceur et cela, particulièrement, le matin du mariage:

13 Ibid., p. 146.

14 Ibid., p. 154. 
Elle aperçut sa mère, lourde, qui allait et venait avec peine; et une vision d' elle-même ainsi déformée s'implanta dans son esprit. [...] Oh!, qu'elle haïssait le piège dans lequel elle était tombée! [...] Une expression de refus, presque de haine s'épandit sur ses traits. (p. 344)

Ce n'est qu'après la conquête de «l'estime de soi» qu'apparaîtra, dans la finale du roman, la pensée de l'enfant, et, encore, sera-ce sans débordement, avec réalisme, avec lucidité:

Et graduellement, elle en vint d penser à son enfant, et sans grand ressentiment. [...] Elle ne l'aimait pas encore, cet enfant qui la ferait souffrir, sans doute ne l'aimerait-elle jamais, elle le redoutait même encore, mais elle s' habituerait peu dे peu d̀ le détacher de sa faute de elle, de sa grave erreur. (p. 384)

La trajectoire romanesque du corps féminin se dépouille des clichés et stéréotypes qui l'encombraient. Amaigrissement qui ne retient que l'essentiel.

Le rapport de Florentine à l'objet alimentaire témoigne encore de son dédain pour l'embonpoint. Chez elle, aucune appétence pour les substances nourricières, aucun signe de gourmandise qui s'expliquerait pourtant aisément du fait de l'extrême dénuement de la famille. L'acte de se nourrir est toujours pour autrui, toujours orienté vers l'axe du devoir: soit que la jeune serveuse, dans l'obligation de gagner sa vie, assure le passage des mets en échange d'une maigre pitance ou de ces restes que sont les pourboires, soit qu'elle offre, dans un moment d'oubli de soi, un bon repas à sa mère (déjà grosse), soit qu'en guise d'appât, elle prépare du sucre à la crème pour Jean.

Ce n'est certes pas la bonne chère qui éveille la convoitise de la jeune fille lors de ses deux sorties au restaurant en compagnie de Jean, mais ce qui lui met l'eau à la bouche, c'est bien plutôt l'élégance du lieu, son décor de bon goût, soit la blancheur des nappes, le miroitement des cristaux (p. 81), l'éclairage discret, bref la transparence du lieu qui est à l'image de son corps désincamé. Le désir manifeste de Jean d'engraisser celle qui n'a que la peau et les os - lui donnant un pourboire: Et qu' il te serve d manger quelque chose de nourrissant, th es bien trop maigre (p. 25), ou encore, l'ayant invitée au restaurant, il souhaite la voir manger au moins une fois d sa faim (p. 83) -, ce désir, qui n'est pas sans analogie avec celui de l'ogre et de la sorcière, forme un saisissant contraste avec la tendance anorexique de Florentine qui touche à peine aux mets variés et appétissants qui se succèdent sur la table, se contentant de picorer dans son assiette comme un oiseau, trop surexcitée pour trouver une saveur aux aliments (p. 83) ou encore ne faisant que porter une fourchette d̀ sa bouche pour la laisser aussitôt retomber (p. 189). On comprend le malentendu profond qui les sépare et qui provient de l'incompatibilité des codes sexuels auxquels ils adhèrent: ici, l'appétit masculin réclame son dû, une chair appétissante; là, l'inappétence féminine tente de se soustraire à l'impératif des hanches larges. La maigreur se vit donc dans l'ambiguité. Car l'acte de se nourrir relève doublement d'un instinct vital 
primordial: si sa principale fonction est de sustenter le corps, il alimente aussi l'imaginaire qu'il soutient et enrichit. En ce sens, l'indifférence de Florentine pour la matière nourricière - qui se mue en dégoût lorsqu'elle assiste au spectacle quotidien de la mastication - l'anémie et la maintient dans un état de privation existentielle:

De biais, elle voyait plusieurs visages ramassés sur des assiettes, les bouches ouvertes, des mâchoires mastiquant, des lèvres grasses - un spectacle qui toujours l'irritait profondément. (p. 20)

Mais la contrepartie ou l'option proposée est-elle plus enviable? Cela donne la grosse Marguerite, le négatif de Florentine, toujours friande de grasses nourritures. À deux reprises, ce personnage matemel est associé au mets riche et crémeux qu'est le sundæ. Une première fois, dédaigneusement, par Florentine qui l'imagine comparant la vie d̀ une perpétuelle ronde de sundas (p. 19). Une deuxième fois, longuement décrite par l'instance narrative dans l'acte délectable de la préparation des riches et onctueuses matières qui composent le sundæ:

Elle levait un couvercle, plongeait la cuiller creuse dans l' épaisseur de la crème glacée... Elle ajoutait un peu de crème fouettée en la faisant couler... elle puisait une cuiller de guimauve blanche et la faisait ruisseler sur la crème, elle arrosait le tout de caramel ou de sirop et plaçait enfin au sommet une demi-cerise confite, rouge et appétissante. [...] Le procédé ne variait guère, mais Marguerite mettait autant de soin et autant de joie naïve à construire le beau et savant édifice d' un dixième que d' un premier sunda. (p. 18)

On le voit, le plaisir suscité par les mélanges de crèmes et de glaces ruisselantes n'est pas sans évoquer une autre sorte de jouissance. De même que son inlassable capacité de répétition. Or, se souvenant que Marguerite a pour fonction de symboliser la maternité euphorique, le lecteur a vite fait d'établir l'équation: appétit $\rightarrow$ corps plantureux $\rightarrow$ procréation. C'est cette équation que Florentine cherche à déconstruire pour la retourner comme un gant: inappétence $\rightarrow$ maigreur $\longrightarrow$ infécondité.

En effet, non seulement est-elle présentée comme indifférente aux voluptés de bouche, mais aussi peu tentée par le plaisir sexuel. Pas le moindre soupçon de désir n'effleure son corps que le texte ne décrit jamais comme ému par la proximité du corps masculin. Le mot d'allié (p. 22) plutôt que celui d'amoureux ou d'ami est choisi pour désigner le genre de relation qu'elle veut entretenir avec Jean. Et si l'envie la prend de saisir à pleines mains ses cheveux drus et forts, ce n'est pas par attirance sexuelle, mais bien pour en recevoir, comme par magie, les vertus de force et de détermination qui s'en dégagent:

Elle aimait la façon dont ses cheveux noirs, abondants se dressaient tout droits et hérissés. Elle avait par instants le goût de saisir ces cheveux forts et sauvages a pleines mains. (p. 16) 
Son envie de saisir cette chevelure fortement sexualisée ressemble à «l'envie du pénis», non selon Freud, mais tel qu'interprétée par Simone de Beauvoir dans le Deuxième Sexe:

En ce qui concerne la femme, son complexe d'infériorité prend la forme d'un refus honteux de sa féminité: ce n'est pas l'absence du pénis qui provoque ce complexe mais tout l'ensemble de la situation, la fillette n'envie le phallus que comme le symbole des privilèges accordés aux garçons; la place qu'occupe le pere dans la famille, l'universelle prépondérance des mâles, l'éducation, tout la confirme dans l' idée de la supériorité masculine. ${ }^{15}$

En effet, ce dont veut se saisir l'être carencé, ce n'est certes pas la chevelure en elle-même ni le sexe viril qu'elle symbolise, mais bien les attributs de puissance et de liberté qui lui sont métonymiquement conférés. Enfin, la scène pathétique du divan illustre avant tout l'imprudence et l'ignorance qui l'ont conduite à s'abandonner à la voracité de l'autre plutôt qu'elle ne représente la soumission à une jouissance envahissante et irrépressible. En dernier ressort: échec pitoyable de la maigreur qui n'arrivera ni à éviter la grossesse ni à être assez alléchante pour satisfaire pleinement l'appétit de l'ogre qui ne peut se contenter de cette friandise qu'est le sucre à la crème ou de cette fin de repas qu'est le dessert:

Au dessert, il vint s'asseoir près d' elle. Il enserra le poignet de la jeune fille de sa main; il semblait en mesurer le tour; il enfonçait ses doigts autour des petites veines gonflées; il regardait ce poignet meurtri comme fasciné; puis il remonta en suivant l' intérieur du bras jusqu' au coude. Alors sa main se raidit sur la chair tendre. Elle sentit sa main qui la bralait. ${ }^{16}$ (p. 189)

À la brûlure de la main masculine, Florentine répond par des paupières qui s'alourdissent, par le rêve: [...] et elle fut alors dans une brume qui, de partout, se refermait sur elle (p. 189). La sexualité, zone blanche, muette, impensable, place la femme à contre-courant et de la réalité et des sources d'énergie libidinale. L'éros impitoyablement refoulé laisse s'engouffrer les forces destructrices qui dévorent l'être et le privent de l'élan vital nécessaire au triomphe de l'individualité. Florentine n'a à son actif, pour toute énergie et pour toute défense, que sa maigre maigreur.

\section{Mère-mort}

Le dévidage de l'écheveau romanesque conduit le jeune corps nubile jusqu'au terme inéluctable, le corps fécond. On a vu que l'introduction du personnage de Rose-Anna a lieu bien après celui de Florentine, au moment où celle-ci va au devant du danger encouru par son sexe. Ce déroulement traditionnel se veut

15 Simone de Beauvoir, op. cit., p. 58.

16 Je souligne. 
mimétique de celui qui gouverne l'existence d'une femme. Mais par une sorte de bizarrerie du temps, comme s'il se détraquait, au lieu de l'image de l'épanouissement et de l'avenir, une régression s'opère et c'est une figure maternelle épuisée, accablée, en danger de mort, qui fait son apparition. Vision propre à inspirer l'effroi et qui fait fonction de présage.

En effet, c'est dans une position mortuaire que Rose-Anna est présentée pour la première fois: [...] étendue sur son lit, au fond de la pièce double, elle a les paupières closes, les mains abandonnées sur sa poitrine (p. 69). Comme si elle s'ajustait à l'espace étroit qui lui est chichement alloué depuis si longtemps, elle est à peine visible dans l'ombre où elle [est] livrée sans défense (p. 69). Livrée tout ce temps aux pensées d'effroi, de menace et de malheur qui rôdent autour d'elle comme la mort elle-même. C'est donc dérisoirement un corps cadavérique que Gabrielle Roy prête à celle qui est porteuse de vie, comme si elle avait été dévorée vivante par sa progéniture:

Son visage parut terreux, avec de grands trous d'ombre à la place des yeux. Des mèches tombaient en désordre sur ses joues et ses lèvres remuaient sans qu'il en sortît aucun son. Elle semblait vieille soudain et prête à s'écrouler. (p. 75)

La mort semble s'être installée en elle, ne laissant de vivant et de productif, par un tragique revirement, que le ventre proéminent qui, en définitive, ne produit lui aussi que des morts en sursis, enfants chétifs et malingres comme le petit Daniel. Tout le reste du corps, au fond inutile, se défait et s'effrite: joues amaigries [où coulent] des larmes vertes (p. 76), voix à demi éteinte ou molle (p. 75), pas chancelants ou ralentis (p. 118), et partout des marques si évidentes de fatigue et d'usure (p. 91). Non seulement cette vision de la décrépitude de la mère est-elle constante, mais elle est encore soutenue, réitérée par des effets de miroir. Témoin cette scène où, Rose-Anna, en proie au désespoir, aperçoit au pied de son lit, lui faisant pendant, une Mère des Douleurs [qui] offrait son cœur transpercé au rayon blafard qui se jouait entre les rideaux (p. 76). Si Gabrielle Roy prend soin de montrer que le corps usé de Rose-Anna recèle des sources d'énergie insoupçonnées, c'est toujours par rares éclairs, brefs et vite disparus, faisant par là la preuve que l'être personnel, individuel a été assassiné par le corps social, au service de la reproduction. C'est autrefois qu'elle avait été gaie, avec des yeux tendres et veloutés, une voix chaleureuse (p. 149). La distinction est de la sorte constamment maintenue entre la personne et la fonction. Et pour que pareil effondrement ne soit pas attribué à la seule pauvreté, l'auteur verra à fournir un portrait contrasté de l'homologue masculin. Azarius était resté jeune, beau de sa santé inaltérable, alors qu' elle montrait des marques si évidentes de fatigue et d' usure (p. 91).

Il arrive plus d'une fois, dans le parcours romanesque, que surgisse dans le champ de vision de la fille un corps de mère inacceptable, souffrant et diminué. La réaction immédiate est le rejet de celle qui émerge comme pôle identificatoire. Il devient dès lors évident que l'objet en litige entre mère et fille est bien le corps 
reproducteur. Songeons à la visite inopinée de Rose-Anna au Quinze-Cents alors que Florentine atterrée a la révélation soudaine de la dégradation du corps-objet:

Sa mère! ... Elle lui semblait bien vieille. Elle se mouvait avec lenteur et son manteau trop serré lui faisait un ventre saillant. (p. 124)

Cette découverte stupéfiante la conduit à consommer, du moins dans son esprit, la rupture avec le modèle proposé:

[...] et c'était comme si, aujourd' hui, elles eussent en quelque sorte à se faire des adieux. Peut-être ici même leurs routes étaient-elles en train de se séparer. (p. 120)

On se souvient aussi que le matin de son mariage, la vision de la mère lourde et déformée la remplit d'une atroce indignation (p. 344). Mais la scène où le ventre porteur apparaît comme le nœud insolvable est celle où les deux femmes enceintes se dressent l'une contre l'autre comme deux ennemies. Situation tragique que celle où une mère, découvrant la grossesse de sa fille, reporte sur celle-ci la haine et l'horreur éprouvées pour l'humiliation et la honte du corps captif. Et, dans le même temps, dans un terrible face-à-face, comme dans un miroir, le regard de la fille se porte sur un corps de mère négligé, dans une robe poussiéreuse et les cheveux défaits (p. 262). Une indistinction expressive entre mort et enfantement s'établit alors que les épaules de la mère vont et viennent dans un balancement ininterrompu, triste et monotone, comme si elle berçait un enfant (p. 262), alors que le creux de sa robe, entre les genoux, la courbe de ses bras qui semblaient soutenir un poids (p. 262) rappelle à Florentine le petit Daniel mourant que la mère calmait de cette façon. Â la belle image du sein maternel, des entrailles bénies, se substitue celle, moins flatteuse, d'un corps en déroute, semblable à ces caisses éventrées et ces armoires boiteuses (p. 259), qu'amène dans cette même scène cette invasion de leur logis par une troupe d'étrangers (p. 260).

L'identification mère-mort va aller en se précisant pour culminer dans une coïncidence frappante par laquelle se trouvent réunies, dans un même chapitre, l'arrivée du nouveau-né et la disparition de Daniel, l'enfant atteint de tuberculose: Qui donc était mort? Daniel? le petit enfant? (p. 361). De multiples images antagonistes se soudent entre elles, amenuisant, jusqu'à sa disparition, l'espace entre la naissance et la mort: 1) un petit cercueil blanc [...] mince cercueil, à peine plus grand qu' un berceau; 2) un enterrement, un baptême [...] prenaient à ses yeux le même caractère tragique, insondable, amer; 3) Rose-Anna aperçoit tantôt une fosse fraîchement creusée, toute prête pour le petit cercueil et tantôt un enfant endormi dans sa longue robe de baptême (p. 361). Dans le texte, les images conjuguées de la naissance et de la mort ramènent invariablement au corps de la parturiente, tel que perçu par celle qui accouche pour la douzième fois et qui a honte d'avouer les douleurs de son corps, qui en a peur, une vraie peur (p. 361), qui redoute un peu plus chaque fois, la honte de cette nouvelle soumission a la douleur (p. 362), mais qui se plie à l'atroce emprise de la douleur 
(p. 363). C'est ce corps des douleurs qui définit de nouveau la filiation mère-fille dont il dessine du même coup les limites:

Mon Dieu, Florentine était mariée. Un jour, elle aussi serait abandonnée d la souffrance et d l' humiliation du corps... [...] Toujours, elle avait voulu des filles. Et pourtant, au dernier moment, elle avait désiré mettre au monde un enfant mâle qui souffrirait moins qu' elle. Toujours, dans l'obscurité, dans la solitude, a travers la détresse du corps, elle avait redouté de donner naissance d une fille. (p. 362)

Pour la première fois, dans les annales romanesques québécoises, l'acte de l'accouchement n'est pas escamoté ou occulté, mais il est, au contraire, mis en scène et ce, sans embellissement. La douleur et l'humiliation ressenties reçoivent un éclairage direct et prolongé d'une violence telle que le corps-mort finit par supplanter le corps-mère:

Dans un effort surhumain, elle se redressa, elle repartit vers sa chambre, elle tomba sur son lit et elle se vit, soudain, couchée d son tour dans un cercueil, un chapelet autour de ses mains qui étaient devenues toutes paisibles, tout unies. Un si grand besoin de s'en aller ainsi dans la mort, de se dérober d toute souffrance la saisit qu' elle noua ses mains sur sa poitrine pour ressembler d cette vision si douce qu'elle avait d'elle-même. (p. 362-363)

Il ne s'agit pas d'une vision pessimiste, mais d'un regard jeté de plein fouet sur la réalité des accouchements successifs, vécus dans des conditions quasi inhumaines. La brève description de l'enfantement comme source de rajeunissement et d'émerveillement (p. 366) récuse l'objection d'une outrance de vue et atteste, au contraire, de la foi dans les forces vitales. Mais, de par sa brièveté même, elle en exprime la problématique réalisation. Entre la reproduction en série et la mort, toutes les deux incontrôlables et proliférantes, il n'y a plus de différence. Du même coup, le corps de la femme, qui s'enlise répétitivement dans la matière, fait apparaître son identification au chaos et au manque.

Le roman se clôt significativement sur une nouvelle scène corporelle qui réconcilie en une vision commune les corps, jusque-là antinomiques, de Jean et de Florentine. Elle, bien mise avec sa jolie robe de soie imprimée, ses fins souliers, son sac de suède (p. 382-383), l'aperçoit lui pareillement soucieux de son apparence, vêtu d'un complet neuf de bonne coupe, sa cravate de même teinte que les souliers d'été et portant un chapeau de feutre souple (p. 382). De cette description ont été éliminées les marques physiques typiques et du sexe fort et du sexe faible de manière à ne retenir que les seules marques extérieures du succès remporté grâce à la ténacité et à la ruse. Cette coïncidence finale entre deux images corporelles naguère antagonistes préfigure la proche décision de Florentine de se soustraire à l'emprise du regard masculin pour accéder à l'autonomie, ce qui lui vaudra d'enfin connaître une satisfaction qu' elle n'avait jamais éprouvée, l'estime de soi (p. 383). Ainsi, du début à la fin, la trajectoire des corps roma- 
nesques trace-t-elle la voie de l'émancipation féminine comme ne pouvant s'atteindre que dans une entière dé-réification de la femme.

L'éclairage puissant que Gabrielle Roy jette sur le corps féminin dans Bonheur d'occasion crée un effet de réel idéologique des plus efficaces en ce qu'il est sans cesse implicitement rappelé au lecteur l'irrémédiable subordination de la femme à son corps dans un monde où sa fonction sexuelle spécifique est considérée comme un destin biologique, concept qui se trouvait encore renforcé à l'époque, au Québec, par les impératifs de la foi et de la perpétuation de la race. Éclairage qui contribue aussi à libérer le corps textuel féminin tel qu'il avait été figé par une tradition littéraire québécoise. Par la suite, Gabrielle Roy procédera, non plus par le négatif et le manque, mais par le positif et le plein. L'inscription du corps se fera dès lors plus rare car, dans sa galerie de personnages, la procréatrice aura cédé la place à la créatrice. 